the cases in which an abscess has formed in convexion with the appendix, and yet while it is pretty obvious that the anterior abdominal wall has not become attached having felt great anxiety that the abscess might burst into the general cavity, I have opened the abdomen, found a quantity of serous or perhaps sero.purulent fiuid free and a mass of matted bowel in the iliac fossa. In such cases I have wrapped the matted mass up in sterilised iodoform gauze and differentiated the several layers of the abdominal wall with sutures. This gauze I removed within a variable time. In some cases on its removal pus escapes from within its folds which have shut off the general peritoneal cavity by their presence, while in most the mass has subsided considerably in bulk. In the latter case the opening in the abdominal wall was closed at once. I need hardly point out the safety of this measure $A$ surgeon may readily hesitate to expose the patient to the risk of opening an appendical abscess, removing the appendix and draining through healthy peritoneum, especially if the assistance at his command is not of the best; but these means I have cudicated secure the patient's life at a minimum of risk from operative interference.

There is little to say about the treatment of such cases of intense peritonitis as result from the rupture of an abscess in commanication with the interior of the appendix into the general peritoneal cavity or by the establishment of a direct sommunication of the interior of the appendix with the general carity except that interference to be of any Fervice must be very prompt. I am much opposed to lushing the peritoneum in these as in all other similar conditions, but prefer to wipe the parts thoroughly and then, if there is not much distension of the intestines, to wrap them up in a quantity of sterile guze. This causes no serious damage to the peritoneal covering and can be removed readily later. It is very important that plain sterile gauze should be used for this purpse and not a gauze containing any irritating germicidal substancs. In my experience a large number of these conditions are brought about by violence. The appendix is anchored by a recent or old abscess to the posterior wall of the abdomen and the traction exerted on it by the sudden and violent displacement of viscera results in the general infection of the peritoneal cavity.

\section{TWO CASES OF INTESTINAL RESECTION FOR CANCER AND ONE CASE OF "INTESTINAL EXCLUSION." 1}

\section{BY C. B. K E E T L E Y, F.R.C.S. ENG.,} SERGEON TO THE WEST LONDON HOSPITAL.

CASE 1. Intestinal exclusion in a case in which an artificial anus opened into a coil of small intestine, which, in turn, comenunicated by a fistulous track with the rectum.-I saw a ryoung woman aged seventeen, a patient of the late $\mathrm{Mr}$. Bruce Payne, on Jan. 25th, 1894. Her illness began a year previously. Pus had been escaping per anum and the temperature had been hectic all the time. The illness had followed exposure of the feet and legs to cold and wet. On rexamination I found that the hypogastrium was the seat of an abscess on the point of bursting, the skin being blue and thin, and an aren of cerema spreading widely around it. With $\mathrm{Mr}$ Payne's help and that of Dr. Trethowan, I incised the parts freely and followed ap the suppurding tracks wherever they had burrowed both in the abduminal wall and $p$ lvic cavity. The pus was freal and one track led into the rectum. The uterus and its appentages were held stiflly in one position, the $u^{+}$erus over to the right, by inflammatory thickening in the broad ligament The right Fallopian tube felt hard, thick, and convoluted, but the oraries seemed normal and not aduerent peritoneally. A minutely careful process of scraping and sponging was gone through, requiring free incisions, two of which divided the recti over the pubes. My experience is that mere drainage is all but useless in such cases. The peritoneal cavity was opened, but not untıl the cleansing process was finished. It could

1 A paper read at a meeting of the Melical Society of London on a.t. $23 \mathrm{th}, 1895$. not be closed again as the hypogastric parietal peritoneum and sub-peritoneal tissue were too soft to hold sutures. Drains were arranged, sutures passed through the skin, aponeuroses, and recti. An aperture still remained, leading into the peritoneal cavity, which was closed with iodoform gauze. The wound was dressed night and day on the following day (Fiday) and again on the Saturday morning by myself. A fortnight afterwards I saw the patient again. Her general condition was very good, but a regular false anus leading into the small intestine was developed at the bottom of the wound. I recommended the recesses of the wound to be washed out with iodıne water and once a week with nitrate of silver ( $5 \mathrm{gr}$. to $1 \mathrm{oz}$ ) and an occasi nal injection of iodoform in oil. When I next saw this patient, nine months afterwards, the fæcal fistula leading into the small intestine was large enough to admit a hen's egg with ease, and from it another fistula about three inches long led straight into the rectum. It would be wearisome to relate how I tried to remedy things by plastic operations with very little benefit. There was no difficulty in keeping the temperature down and the general condition good in a nursing home in London, but in spite of the sea air the patient always had some degree of hectic at home, though nothing like what had sapped her health before the original operation. Finally on April 17th, 1895, the following operation of intestinal exclusion was performed. Mr. Lloyd administered the anæsthetic and $\mathrm{Mr}$. Scott Elliot and Mr. H. Wharry assisted me. Measures were taken to keep the patient warm. The ileo-rectal fistula was thoroughly cleansed. The incision was semicircular and transverse through the recti. She was now placed in a semi-Trendelenberg's position. I separated the intestinal and mesenteric adbesious to the abdominal wall near to the fæcal fistula, care being taken not to tear those immediately around the fistula, half an hour being thus occupied. The condition then existing is shown in the annexed diagram. Four constricting rubber tubes were

FIG. 1.

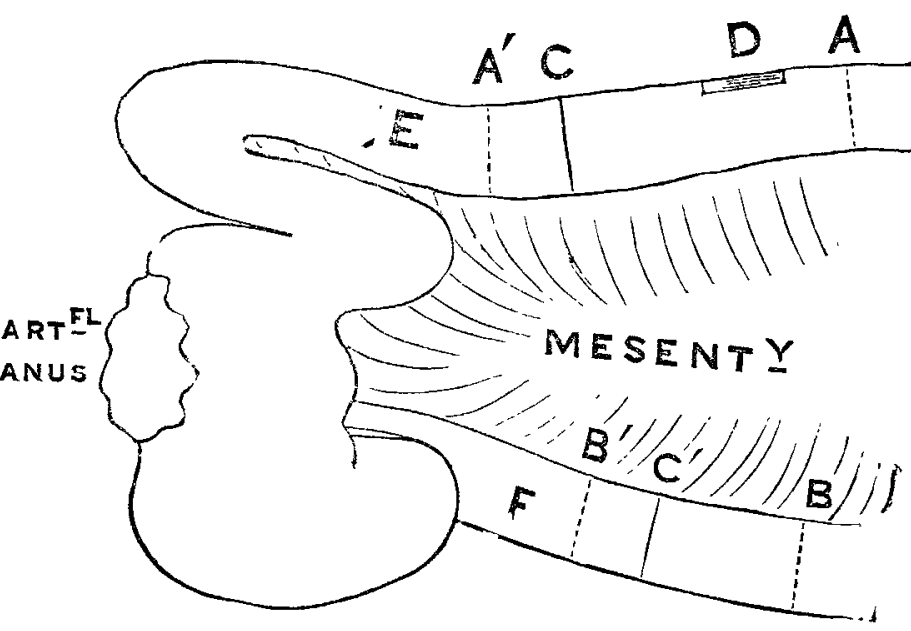

Diagram illustrating Case 1 . By mistake it represents a view from below, not from above, as the intestiue was actuall from below, not from above, as the intestiue was actually to the left side of the abdomen.

applied at $A . A^{\prime}, B, B^{\prime}$ respectively, and a short longitudinal slit made at $D$. Transverse division at $C$ and $\mathrm{C}^{\prime}$ permitted suturing of $\mathrm{C}$ to $\mathrm{C}^{\prime}$ to be performed by Maunsell's methodi.e., after both ends were intersuscepted through the segment $\mathrm{C} A$ and out at the slit D. Silkworm gut sutures were used. The excluded ends were then sewn up. The part $\mathbf{E}$ was drawn inside out through the artificial anus and then sewed up. On returning it, however, I could not satisfy myself that it was securely closed, so I did this part of the proceedings over again. The same half-closed appearance again presented itself, but I then found it was only apparent. The part $F$ could not be turned inside out and was closed from the peritoneal aspect. No drainage was used. The patient's recovery was interrupted by the formation of a temporary fæcal fistula which burst out a fortnight after the operation and was only persuaded to close after long treatment by injections of various kinds, by the electro-cantery, and by a starvation diet in which the patient was partly fed by peptonised food placed in the blind intestinal pouch, which still exists and discharges a little mucus or mucopus-very little-above the pubes. It is admirably adapted for physiological experiment. Ultimately I intend to close it by some plastic procedure. The intestino-rectal fistula 
was treated by daily rectal injections of alum water and by an occasional electro-cauterisation, and has long ceased to permit frecal matter to escape, but still discharges a slight purulent secretion. This is why I postpone the final plastic procedure. The patient's temperature is normal, her health is good and she gets about, wearing a truss over the open blind intestinal pouch, which is a part-probably about ten inches-of the lower end of the ileum.

The operation of intestinal exclusion has been done before in Germany in cases of irremovable cancer, but never before in a case of this nature. It is, of course, the unclosed fistula leading into the rectum which forbids complete excision of the excluded coil.

CASE 2. Excision of a large, freely movable, malignant tumour involving part of the iransierse colon, the whole of the splenic tlexune and the descending part of the colon, all the omentum except a strip round the greater curvature of the stomach, and also a coil of small intestine.-.The first case in which I ever excised intestine within the abdominal cavity (as distinguished from strangulated and gangrenous hernia) was that of a middle-aged man in the Mest London Hospital with a large perfectly morable tumour in the left flank. It proved to be malignant, involving part of the transverse colon, the whole of the splenic flexure and the descending part of the colon, all the omentum except a narrow strip round the greater curvature of the stomach, and a coil of small intestine. Senn's plates were used. The enterectomy and suturing itself took sixteen minutes. Although the patient was in an advanced state of cachexia and anæmia and was under ether three and a half hours he suffered very little from the immediate shock. He was not permitted to lose any blood; everything was clamped before division, and he was most carefully kept warm. His after-pain was, however, great and continuous, and he died twenty-four hours after the operation. Patients suffering from advanced cancerous cachexia may, as Mr. Rickard Lloyd has pointed out, stand a carefully-conducted long operation well, but fail under the demands made on their strength during recovery, perhaps because the heart muscles are as much degenerated as the voluntary muscles. Mr. Ballance, Mr. Edwards, and Mr. Paget assisted in this case. The only analogous one I know of is that in which von Bergmann removed the pylorus and portions of the attached intestines, the operation lasting four and a half hours and the patient dying three hours after it.

\section{FIG. 2.}

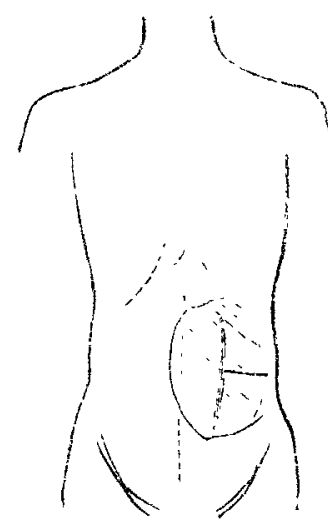

Position of malignant tumour involving large part of colon, omentum, and a coil of small intestine.

CASE 3. Enterectomy for sarcoma near the ileo-ccecal valve; remoral of a large retro-peritoneal glandular round-celled sareoma from the same patient; recovtry: recurrence of glandular tumour and consequent death. - The patient, a girl aged thirteen, was delicate looking, with dark rims under the eyes, and anæmic, and she suffered from pain associated with tumours in the right half of the abdomen, and from symptoms of recurrent partial obstruction, with occasional vomiting. Once a little blood passed per rectum. She was a patient of Dr. Wm. Keen of Chelsea, and was seen by me, and admitted into the West London Hospital for operation, on Feb.19th, 1894. Her condition at that time was as follows. Two distinct, more or less rounded tumours were palpable in the right abdomen, one just to the right of, and above, the umbilicus; the other between the umbilicus and the right Poupart's ligament. The percussion note over both was comparatively dull, and there was some tenderness. The urine was acid, of specific gravity 1020, and turbid, containing a deposit of phosphates, but no albumin. She had been ill for a year, but a lump was noticed five months ago. It had several times seemed to disappear. She had been in bed for the last month. Pain was experienced when walking and about an hour after food. The bowels opened regularly. Cardiac dulness was noted up to third interspace and in the middle of sternum. The apex beat was in the fifth space (?) in the nipple line. The first sound at apex was rough and prolonged. There was a mitral systolic murmur and a presystolic thrill. There was also a double aortic murmur. She had never had rheumatic fever. On the 22 nd the tongue was somewhat dry, and there was a faint trace of albumin in the urine. On the 23 rd the operation took place, which lasted!two

FIG. 3.

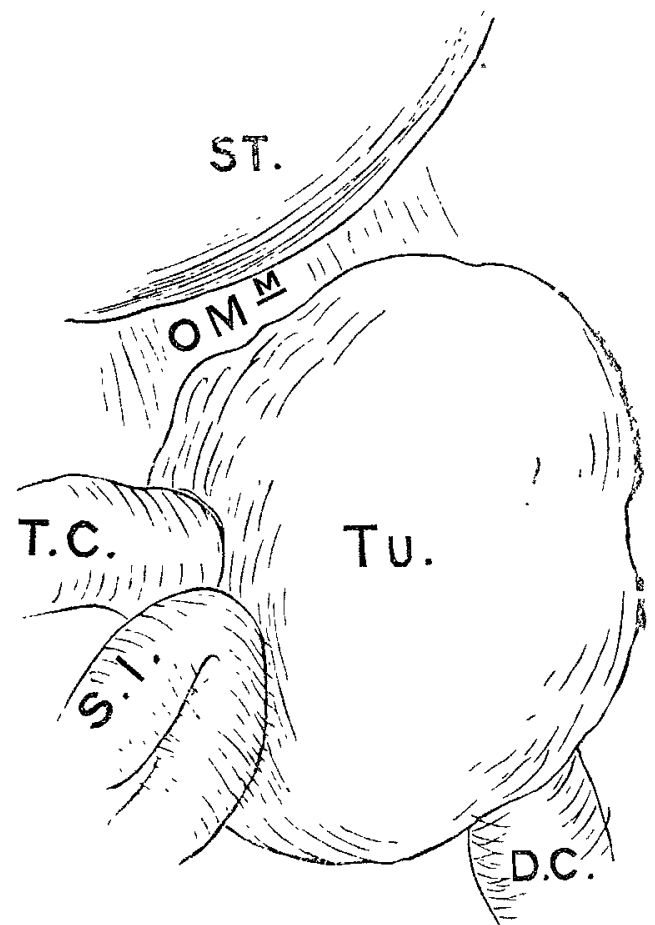

S. F.

Diagram of the tumour itself in Case 2. ST., Stomachi: intestine. D.C., Descending colon. S.F., Sigmoid flexure.

and a quarter hours. Light anæsthesia was induced by $\mathrm{Mr}_{\text {. }}$ Lloyd. The upper tumour being found movable, an incision four and a half inches long was made so as to command both this and the lower tumour-i.e., perpendicularly near the linea semilunaris, and reaching upwards to just above the level of the umbilicus. The abdominal wall was quickly cut through and the tumour and omentum presented immedis ately. The tumour was obviously inside the ascending colon.

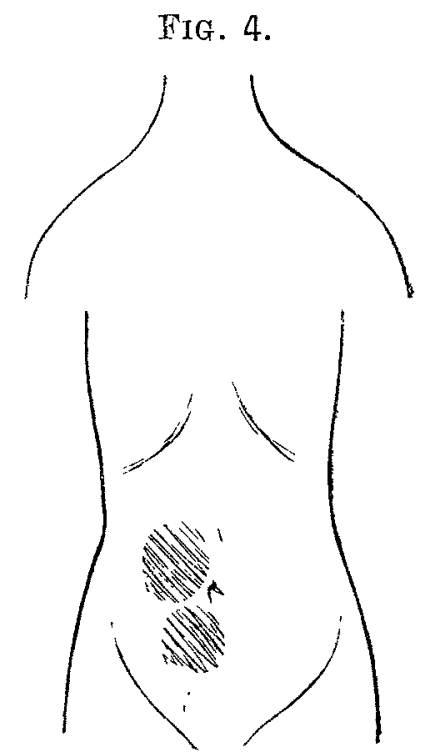

The latter was incised longitudinally, when the tumour was seen as a firm, irregular, congested, fleshy mass smeare with fæcal matter. Its surface had a mucous membrane-like aspect. It was about the size of an orange, its base of attachment being wide. During the rest of the operation, Dr. Joseph Wilks, house surgeon, who was assisting, gave his undivided attention to preventing contamination of the peritoneal. 
cavity, and, having cleaned the tumour, I raised it and with scissors cut through the now stretched intestinal wall which formed its base of attachment all round. The small intestine (intussusceptum) was then seen entering the tumour. This being clamped with a pair of large forceps was cut through, and a few more touches of the scissors permitted the mass to come away. There were now, of course, two apertures in the ascending colon. As the one last madei.e., by cutting round the base of the attachment of the tumour-was much larger than the calibre of the small intestine, the latter was sutured only to the lower part of it by interrupted sutures, and the rest was closed by a continuous suture. The peritoneum was not insluded in these sutures, which were, of course, inserted from within. The original incision in the large intestine - viz., that through which the tumour had been delivered-was closed (1) by continuous suture of the mucous membrane, and (2) Lembert's sutures of the peritoneal and muscular coat. A row of Lembert's sutures was :also inserted into the peritoneal aspect of the junction between the small and the large intestines. The sutures were all of silk, very fine for the peritoneal and of medium thickness for the mucous side. The retro-peritoneal tumour, about the size of a rather large orange and doubtless glandular, was lieft to be removed when the patient had recovered sufficiently A rubber drainage-tube was inserted through an aperture made in the right flank, and a glass tube through one in the linea alba above the pubes. In spite of her anæmic and emaciated state she soon rallied from the anxsthesia and the operation.

The notes of the after-course of the case, kept with the greatest care and ability by Dr. Wilks, are exceedingly full, minute, and valuable, and no abstract can do justice to the picture they present. The temperature usually varied setween $98^{\circ}$ and $99^{\circ} \mathrm{F}$. On the fifth, sixteenth, and thirty-first days it rose to $100.5^{\circ}, 100.8$, and $102^{\circ}$ respectively and on the evening of operation it sank to $97^{\circ}$. The pulse was during the first week from 132 to 140 , during the second from 100 to 116 , and then sank to between 90 and 100 . The bowels were opened nearly every day, frequently twice or thrice $a$ day in the first three weeks, and even six thimes on the fifth day. I suspect that the sutures caused intestinal irritation, as after the larger ones came away on the fourteenth and eighteenth days the stools soon grew less frequent. Blood-stained fluid was removed from the glass pelvic drain for the first three days at frequent intervals, and afterwards serous fluid. Very little pain occurred during the first day or two, but the tongue was rather dry and the face had a pinched aspect. On the fourth day she was not so well. There was a white fur on the tongue. Turbid yellowish tuid came from the pelvic drain. The abdomen was tender and distended and the knees were flexed. On the next day (t the fifth) at 4 A.M. she vomited. A rubber tube was substituted for the glass one, and (on the fourth day) she was fed by nutrient enemata, and only an effervescing mixture and peppermint water were given by the mouth. Whe soon improved. On the eighth and sereral succeeding days the sinus accupied by the pelvic drain was very gently irrigated with warm saline solution. Vomiting and retching recurred occasionally, therefore on the tenth day all food by the mouth was stopped, except Valentine's meat juice diluted, one ounce every four hours. The vomiting soon ceased and her condition improved. For some days she had only the diluted meat-juice by the mouth and beef tea per rectum. On the sixteenth day the enemata were discontinued and two eggs with two thin slices of bread-and-butter allowed. Next day (the seventeenth) there were signs of suppuration beneath the wounds and they were examined. Pus was washed out with the warm saline solution and iodoform powder inserted. At night there was a discharge of pus and of "a thick semisolid substance like hard boiled yolk of egg." On the eighteesth day she was allowed minced chicken. It was on that day that the sutures began to be discharged per anum. The threatened danger of fæcal fistula (?) passed off immediately. A freal smell in the discharge was noticed only on one day. On the twentieth day there was no discharge, and the drainage-tube, which had already been shortened, was discontinued. She was now free from pain and on ordinary diet. The tongue was clean, the appetite was good, she "felt well," and the temperature was normal. Unfortunately, however, the glandular tumour had greatly increased and extended now on the left as far as the median line and upwards nearly to the umbilicus. This was operated on on
March 30th, five weeks after the enterectomy (a week's delay being due to an accidental rise of temperature on March 20th, probably due to confined bowels). The following are Dr. Wilks' notes :-

'On March 30th, 1894, operation under ether was performed by Mr. Keetley ; anæsthesia was first obtained by chloroform. An incision nearly six inches in length was made just to the right side of the umbilicus, its upper extremity being a little above that point. On cutting through the abdominal wall and after the stopping of all bleeding points the peritoneum was exposed. It was thickened and adherent. The tumour felt cystic and an aspirating needle was put in, but no fluid escaped. The peritoneum was opened and the tumour exposed, when it was found to have very firm adhesions to a large area of the abdominal wall (from the umbilicus to Poupart's ligament) and also to the great omentum, which was itself greatly thickened either by inflammation or by extension of the growth. These adhesions were partly torn and partly cut after being clamped with artery forceps. The tumour was also as far as possible enucleated with the finger, but owing to the toughness of the adhesions the scissors also had to be repeatedly used. The intestines gave very little trouble, many coils being collapsed. The close relationship of the tumour to the right ureter, to the right iliac vessels, and to the line of union of the intestinal suture done five weeks ago was a greater source of anxiety. Besides the tumour itself a considerable quantity of diseased omentum was removed. No ligatures were applied, but the bleeding was stopped by forcipressure or by twisting. The site after the removal of the tumour formed a large cavity, the walls of which tended to bleed at many points in spite of sponge pressure. It was packed with strips of iodoform gauze moistened with weak sublimate solution. No drainage-tubes were inserted. The incision was closed-all but a small portion for strips to project-by silkworm-gut sutures. Iodoform gauze and wood-wool pads were applied. The removal of the tumour occupied thirty-five minutes and the whole operation fifty-five minutes. The patient was a little collapsed after the removal of the tumour but soon recovered. At 11 P.M. she had vomited several times since the operation; she was in considerable pain. The pulse was regular, 108, and fairly good. The temperature at 6 P.M. was $97.2^{\circ} \mathbf{F}$. There was no sign of any hæmorrhage. At 11 P.M. the temperature was $96.8^{\circ}$. At 3 P.M. the gauze packing was remove्e from the cavity left by the excision of the tumour ; there was no discharge. She was in considerable pain. There was no vomiting during the day. The temperature was $101 \cdot 6^{\circ}$. On April 1st there was no pain and she slept well; the pulse was 160 , and the temperature $102.8^{\circ}$. On April 2nd the pulse was 132 , and the temperature $1004^{\circ}$; a natural semi-solid naotion was passed. On April 3rd some epistaxis occurred. On April 4th the temperature was $99.4^{\circ}$; there was no pain or tenderness; the wound was dressed and the whole condition was very good. On April 6th, at 12.45 A.M., there was a fæcal discharge from the wound; but a formed stool was passed per anum and the general condition was very good. The temperature in the morning was $98^{\circ}$, and in the evening $99^{\circ}$. On April 7th the old wound (for removal of intestinal tumour) was re-opened; there was a fæcal discharge from it. On April 8th a slough was seen in the re-opened wound. On April 10th this slough was removed."

The patient continued to feel well and look better. The wound of the last operation closed quickly; but the wound of the original operation continued open as a fæcal fistula and water could be syringed through it from the anus. The fistula, therefore, certainly led into the colon. As the fæcal fistula did not form till six weeks after the enterectomy and as a slough came away eleven days after the glandular tumour was removed I think that the necessarily free division of the vessels going to and from the intestine in removing the large and adherent retro-peritoneal tumour had caused the intestine to slough after it had quite recovered from the enterectomy. Indeed, the second operation was practically an ante-mortem examination which demonstrated the success of the enterectomy. The glandular tumour (a round.celled sarcoma) soon recurred and grew to an enormous size, odistending the abdomen and pushing forward the anterior abdominal wall. The tumour was not painful and there was no ferer. Solid motions were occasionally passed ver rectum although the frcal fistula continued open. Patient at first gained flesh a little and then gradualiy emaciated. Occasionally she had 
attacks of vomiting, and at last she died. There was no necropsy.

kemarks. - In the two cases which recovered from the enterectomy and intestioal exclusion respective'y a fæcal fistula formed and kept open fur a long time in the one in which Maunsell's operation was performed precisely, and threatened to form in what was a modified Mdunsell's operation-namely, suture of the inner cuats from within and of the peritoneal coat from without. In Case 1 the open intestinal pouch proved useful for peptonised feeding, when the patient was starred while the fistula was being persuaded to close with the electro-cautery, \&c. The actual suturing occupied only a small part of the above long operations-not more than a seventh of the time in the longest one I would deprecate the constant recommendation of speed in operating on these cases. What should be recommended are economy of blood and warmth, careful and correct apposition of parts, insertion and tying of sutures, and strict antisepsis. Speed in all kinds of operations comes spontaneously with practice. By inculcating speed one may produce hurrythe worst element of all in a delicate procedure, but dawdling and pauses for idle consultation may be freely condemned. I recommend (1) a clock staring the operator in the face. (2) thinking out the possible turns and requirements of the operation before beginning, and (3) a thorough understanding between operator and anesthetist and occasional brief intercommunication between them as to the patient's state. The first case illustrates the advantages of thoroughness in dealing with pelvic abscesses. When a pelvic abscess has burrowed extensively, mere drainage is apt to lead only to exhaustion from hectic, whether or not the case be septic in origin. With regard to material for intestinal suture, upon the whole I think silkworm gut too stiff, and would prefer fine silk. In Case 3 the glandular tumour appears to have been discovered before the prime mischief. This occurred in another patient of mine and shows how difficult it is to get these cases early enough for thoroughly radical operation. It was striking how little the advanced cardiac mischief in Case 3 interfered with the powers of undergoing the severe operation and rapidly recovering from it.

Grosvenor-street, W.

\section{ON PERIPHERAL NEURITIS IN PREGNANCY. ${ }^{1}$}

BY GEORGE ELDER, M B., M.R.C.P. EdIN.

THE cases of peripheral neuritis recorded bearing a direct relationship to the pregnant state are at present sufficiently rare to be of interest even when they are comparatively mild. The following two cases have recently come under my notice and seem to be worth putting on record.

CASE 1 -A married woman aged thirty-three years was fir.t seen on Dec. 1lth, 1895, when she complained of want of power and tingling in both hands, which had lasted about two months. She had had already eight children, all born healthy. No miscarriages had occurred. In one of her previous pregnancies (seven years ago) she was said to have had "kidney disease" when five months pregnant. She had not at that time any severe symptoms; there had been no fits, nor had there been any condition like the present, and at full term she had been delivered of a healthy child. Never at any time had she had any tingling in the hands or feet or want of power in them. During her present piegnancy she had suffered from nothing further than a sore throat till the present symptoms commenced. The sore throat only lasted a day or two; there were no others in the neighbourhood affected so far as she knew, and, in fact, there was no reason whatever to think that it might have been diphtheria, as she had of ten such sore throats, evidently exacerbations of the granular pharyngitis present. When about six months pregnant she began to be troubled with tingling and feelings of "pins and needles" in her right hand, especially at nights. These got worse, shooting pains began, and then the hand began to feel cold and she could not use it as she was accustomed to. She was often kept awake at night by

1 A paper read before the Edinburgh Obstetrical Society in June, the sensations in it. Soon the left hand began to suffer in the same way and quite lately she had had a numb feeling about the left haunch. On examination she was found to be a healthy looking woman, eight months pregnant. She had slight headaches, but no sickness, vomiting, or affection of ey esight. There was neither albumin nor sugar in the unine. The heart was quite normal. Some granular pharyngitis was present. She did not look alcoholic and declared she was very temperate. Her hands troubled her greatly, the shooting pains and tingling and feelings of heat and cold being a times very severe. She dropped things out of her hands and could not do any fine work. She could grasp kettles, \&cc. which were far too hot for any one else to lift and had no feeling of heat in doing so. The hands were cold; there was no wrist drop. She could grasp pretty firmly with both hands, though she often dropped articles aftex she had taken them up, evidently from affection of sensation. In both hands sensation was dulled, more in the right than in the left, pain, tactile sensibility, and sensation of temperature all being affected. The feet were not affected apparently.. There was no tenderness on pressure along the line of the nerves. She was confined on Feb. 4th, 1896, and had a good puerperium. The symptoms began to pass away immediately after delivery and in from three to four weeks she was quite recovered except for a very slight tingling in the hands.

CASE 2.-A married woman aged thirty-seven years waA seen at her confinement. This was her eleventh child; all of them had been healthy and no miscarriages had occurred. There was no history of syphilis and she was not alcoholic. She had not suffered from sore throat of any kind. No trouble with her urine had occurred. No symptoms of poisoning by lead or other mineraI poison were present. She had never suffered in the same way before. When about sis months pregnant she began to suffer from tingling and want of feeling in her fingers. Paræsthesiæ became severe and loss of feeling greater; she dropped things and she could not knit or do fine work with her fingers. Some weeks before delivery similar symptoms came on in her feet and had been getting worse. On examination there was found to be distinct impairment of sensation both in the hands and feet, most marked on the palmar and plantar aspects, pain and temperature being more markedly affected than touch, though this also was affected. There was no foot- or wrist-drop and the patient could grasp the hands firmly. The plantar refles was nct gone or the knee-jerk. No tenderness on pressure along the line of nerves was present. After delivery the sensations began gradually to trouble her less and three montbs afterwards she was quite recovered except for slight tingling at times in the hands, the objective affections of sensation being quite gone.

There can be little doubt that these were cases af peripheral neuritis, though very mild in type. So far as one could ascertain (and in the second case $h \in r$ history for some years was known) no other cause for the condition beyond the pregnant state could be found. The patients were not alcohclic, there was no trace of syphilis, or of poisoning by lead, arsenic, or other minerdl poison. There was no diphtheria (the slight sore throat in the first case was, no doubt, from the exacerbation of the pharyngitis) and there was no trace of albumin ni sugar in the urine. Influenza, gout, typhoid fever, pneumonia, scc., could all be excluded, and therefore one has to conclude that the peripheral neuritis was the result of the gravid state, a conclusion corroborated by the fact that recovery began as soon as delivery had taken place.

As has been already stated, the recorded cases of peripheral neuritis closely associated with pregnancy are very few. They have mostly been described as a variety of the peripheral neuritis of the puerperium-"polyneuritis puerperalis"-many of which, though only noticed duing the puerperium, are thought by Eulenburg and ather writers on the subject to originate during pregnancy. The subject of puerperal polyneuritis was first thoroughly investigated by Möbins, although previous records by Lever, Imbert-Gourbeyre, Kast. and others were evidently examples of the disease. Writing in February, 1895, Eulenburg was able to collect thirty-eight cases of puerperal polyneuritis (including one or two cases of polyneuritis occurring daring pregnancy) The polyneuritis of the puerperium is now generally divided into two classes :- -1 . The generalised or diffuse form, affect ing both the upper and the lower extremities. Here tbe affection usually resembles in onset and in character a case of ordinary alcoholic polynearitis. It may, however, begin 